die Unmöglichkeit, ein substituirtes Derivat selbst unter den günstigsten Bedingungen zu gewinnen, nur dadurch befriedigend zu erklären, dass das Stickstoffatom, an welchem die Substitution erfolgen soll, tertiär gebunden ist. Wir erblicken in dem eben geschilderten Widerstande gegen die Substitution des Brückenstickstoffs, soweit solche negative Ergebnisse überhaupt Beweiskraft haben können, eine experimentelle Bestätigung der tertiären Natur dieses Brückenstickstoffs and eine werthvolle Stütze der in der oben mitgetheilten Abhandlung befürworteten Formel für das Murexid.

\title{
II. Ueber das Uramil;
} mitbearbeitet von Carl Finckh.

(Eingelaufen am 9. Februar 1904.)

Das von Liebig und Wöhler ${ }^{1}$ ) entdeckte Uramil, dem Baeyer ${ }^{2}$ ) die Constitutionsformel:

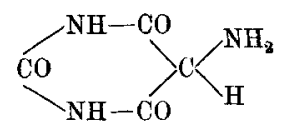

Amidomalonylharnstoff

gegeben, bildet sich beim Kochen von thionursaurem Ammoniak mit verdünnter Schwefelsäure nach der Gleichung

$$
\mathrm{C}_{4} \mathrm{H}_{3} \mathrm{~N}_{3} \mathrm{O}_{6} \mathrm{~S} \cdot\left(\mathrm{NH}_{4}\right)_{2}+\mathrm{H}_{2} \mathrm{O}=\mathrm{C}_{4} \mathrm{H}_{5} \mathrm{~N}_{3} \mathrm{O}_{3}+\left(\mathrm{NH}_{4}\right)_{2} \mathrm{SO}_{4} \text {. }
$$

Es bildet sich ferner neben Alloxan beim Kochen einer Lösung von Alloxantin mit Salmiak. Eine Erklärung dieser sehr merkwürdigen Reaction ist schon in der I. Mittheilung gegeben worden.

Endlich bildet sich Uramil, wie wir fanden, auf sehr einfache Weise, wenn man trocknes dialursaures Ammon (zur Vermeidung von Oxydation der Substanz im geschlossenen

1) Diese Annalen $\mathbf{2 6}, 274$.

2) Diese Annalen 130, 172. 
Rohre) erhitzt. Es wird dabei intramolekular ein Mol. Wasser abgespalten :

$$
\mathrm{C}_{4} \mathrm{H}_{4} \mathrm{~N}_{2} \mathrm{O}_{4} \cdot \mathrm{NH}_{3}=\mathrm{C}_{4} \mathrm{H}_{5} \mathrm{~N}_{3} \mathrm{O}_{3}+\mathrm{H}_{2} \mathrm{O} \text {. }
$$

Durch Auflösen des bräunlich gefärbten Reactionsproductes in Natronlauge und Wiederausfällen mittelst Mineralsäuren kann das Uramil gereinigt werden, jedoch ist die Gewinnung ganz farbloser Krystalle auf diesem Wege schwierig.

Von den Eigenschaften des Uramils hat uns besonders sein Verhalten gegen Alkalien, gegen Oxydationsmittel und gegen Schwefelsäure beschäftigt.

Die Einwirkung von Alkalien auf Uramil wurde schon von Liebig und Wöhler ${ }^{3}$ ) studirt, ohne dass diese Forscher zu einer befriedigenden Erklärung der hierbei sich abspielenden complicirten Vorgänge gelangt waren. Es hat dies seinen Grund wohl darin, dass die Wirkung von Alkali je wach der Concentration und nach der Dauer des Kochens eine sehr verschiedene ist, so dass sich neben einander eine Reihe von Körpern in Lösung befinden. Es entstehen zunächst Salze des Uramils, die dann weiterhin bei längerer Einwirkung von überschüssigem Alkali in sehr interessanter Weise gespalten werden.

\section{Salze des Uramils.}

Das Uramil zeigt durch seine Löslichkeit in Alkalien sauren Charakter; Salze desselben waren aber bis jetzt nicht bekannt. Sie konnten jedoch in grosser Anzahl dargestellt werden und sind sämmtlich wohlcharakterisirt. Die Alkalisalze entstehen durch kurze Einwirkung von Alkali auf Uramil. Dasselbe erscheint in ihnen als schwache zweibasische Säure, welche Salze mit zwei und einem Aequivalent Metall zu bilden vermag. Ausser diesen beiden Typen bildet das Uramil aber noch eine dritte Art von Salzen, in denen auf zwei Mol. Uramil nur ein Aequivalent Base trifft. Aus allen Salzen lässt sich durch Ansäuern unverändertes Uramil wiedergewinnen.

3) Diese Annalen 26, 313. 


\section{Uramildikalium,}

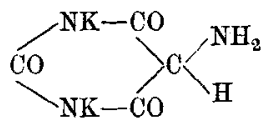

Zur Darstellung dieses Salzes löst man Uramil in der sechs- bis zehnfachen Menge concentrirter Kalilauge $(1: 1)$ unter raschem Erwärmen, wobei in Folge theilweiser Zersetzung sehr starker Ammoniakgeruch auftritt. Wenn sich das Uramil nur mehr träge löst, filtrirt man rasch über Asbest vom unveränderten Uramil ab. Beim Erkalten erstarrt das Filtrat zu einem Gemenge feiner, hellgelber Nadeln. Man saugt ab, wäscht mit kaltem Methylalkohol, bis dieser klar abläuft und bringt rasch in den Exsiccator.

Das Salz ist auf keine Weise umzukrystallisiren, weil die Auflösung in Wasser, in dem es leicht löslich ist, stets mit Kaliumverlust verbunden ist; denn beim Fällen einer wässrigen Lösung 'mit Alkohol oder bei längerem Stehen krystallisiren stets kaliumärmere Salze aus. Es ist daher auch nicht zu entscheiden, ob die gelbe Farbe dem Salze eigenthümlich ist oder von einer kleinen Verunreinigung herrührt. In feuchtem $\mathrm{Zu}-$ stande an der Luft färbt es sich rasch intensiv roth und riecht nach Ammoniak. Das Salz entbält zwei Mol. Krystallwasser, die jedoch sehr hartnäckig festgehalten werden; sie entweichen vollständig bei $150^{\circ}$ im Vacuum unter Blaurothfärbung des Salzes. Auch nach der Entwässerung ist dasselbe vollständig in Wasser löslich.

(I. rohes Kaliumsalz auf Thonteller im Exsiccator getrocknet, II. mit Methylalkohol gewaschen.)

I. $0,1701 \mathrm{~g}$ gaben $0,1190 \quad \mathrm{~K}_{2} \mathrm{SO}_{4}$.

$0,2760 \mathrm{~g} \quad$ " $38,2 \mathrm{~cm}$ Stickgas bei $15^{\circ}$ und $724 \mathrm{~mm}$ Druck.

II. $0,2778 \mathrm{~g} \quad, \quad 0,1887 \mathrm{CO}_{2}$ und $0,0704 \mathrm{H}_{2} \mathrm{O}$.

$0,1976 \mathrm{~g} " \quad 29,5 \mathrm{ccm}$ Stickgas bei $16,5^{\circ}$ und $712 \mathrm{~mm}$ Druck.

$0,2744 \mathrm{~g} \quad, \quad 0,1836 \mathrm{~K}_{\mathrm{g}} \mathrm{SO}_{4}$.

$0,3848 \mathrm{~g}$ verloren im Vacuum bei $150^{\circ} 0,0527 \mathrm{~g}$. 


\begin{tabular}{lccrr} 
& Berechnet für & \multicolumn{2}{c}{ Gefunden } \\
& $\mathrm{C}_{4} \mathrm{H} \mathrm{N}_{3} \mathrm{O}_{3} \mathrm{~K}_{2} \cdot 2 \mathrm{H}_{2} \mathrm{O}$ & I. & \multicolumn{1}{c}{ II. } \\
$\mathrm{H}$ & 18,82 & - & 18,53 \\
$\mathrm{H}$ & 2,74 & - & 2,83 \\
$\mathrm{~N}$ & 16,47 & 15,63 & 16,49 \\
$\mathrm{~K}$ & 30,58 & 31,65 & 30,04 \\
$\mathrm{H}_{2} \mathrm{O}$ & 14,11 & - & 13,69
\end{tabular}

Durch doppelte Unsetzung mit Schwermetallsalzen entstehen amorphe Niederschläge, von denen die mit Chlorbaryum und Bleinitrat allmählich krystallinisch werden. Die Lösung des Salzes giebt mit Mineralsäuren den charakteristisch flimmernden Niederschlag von Uramil.

$0,1235 \mathrm{~g}$ gaben $32,3 \mathrm{ccm}$ Stickgas bei $16,5^{\circ}$ und $708 \mathrm{~mm}$ Druck.

Berechnet für

Uramil

$\mathrm{N}$
Gefunden

28,73

Jod führt das Salz in Murexidkalium über. Mit etwas mehr als zwei Mol. Jodmethyl kalt geschüttelt, entsteht reichlich ein methylirter Körper, in feinen, biegsamen Nadeln krystallisirend, die in kaltem Wasser schwer, in heissem leicht löslich sind. Nach Analyse und Eigenschaften ist er mit dem von T echow ${ }^{4}$ ) beschriebenen 1,3-Dimethyluramil identisch.

$0,1747 \mathrm{~g}$, exsiccatortrocken, gaben $0,2700 \mathrm{CO}_{2}$ und $0,0878 \mathrm{H}_{2} \mathrm{O}$.

$0,1412 \mathrm{~g}, \quad " \quad$ " 32,3 cem Stickgas bei $15^{\circ}$ und $710 \mathrm{~mm}$ Druck.

$\begin{array}{ccr} & \text { Berechnet für } & \text { Gefunden } \\ & \mathrm{C}_{6} \mathrm{H}_{9} \mathrm{~N}_{3} \mathrm{O}_{3} & \\ \mathrm{C} & 42,10 & 42,16 \\ \mathrm{H} & 5,66 & 5,62 \\ \mathrm{~N} & 24, \tilde{5} 6 & 25,33\end{array}$

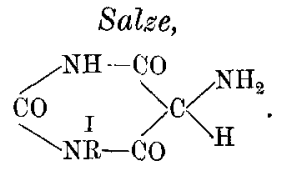

Alkalisalze von diesem Typus konnten nicht erhalten werden, dagegen ein Blei- und ein Barytsalz.

4) Ber. d. deutsch. chem. Ges. 27, 3088. 
Giebt man zur klaren Lösung von Uramil in Ammoniak Chlorbaryumlösung, so fällt sofort ein schweres, durch Oxydation schwach rosa gefärbtes Barytsalz aus.

$0,1639 \mathrm{~g}$ gaben $0,0902 \mathrm{BaSO}_{4}$.

$0,1573 \mathrm{~g} \quad, \quad 0,0874 \mathrm{BaSO}_{4}$.

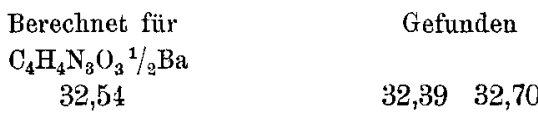

$\mathrm{Ba} \quad 32,54 \quad 32,39 \quad 32,70$

Das Bleisalz erhält man, wenn man Uramil in der Wärme in einer bekannten Menge Natronlauge löst und dazu eine heisse Lösung von Bleiessig giebt, die mit so viel Essigsäure versetzt ist, als zur Neutralisation der angewandten Natronlauge berechnet ist. Es fällt dann sofort ein sehr schwer lösliches, durch Oxydation gleichfalls rosa gefärbtes Salz aus. Aus der Mutterlauge krystallisiren nach längerem Stehen noch geringe Mengen feiner Nadeln des Salzes.

$0,2773 \mathrm{~g}$ gaben $0,1715 \mathrm{PbSO}_{4}$.

$\begin{array}{cc}\text { Berechnet für } & \text { Gefunden } \\ \mathrm{C}_{4} \mathrm{H}_{4} \mathrm{~N}_{2} \mathrm{O}_{3}{ }^{3}{ }_{2} \mathrm{~Pb} & \\ 42,16 & 42,23\end{array}$

$$
\text { Salze, } \mathrm{C}_{5} \mathrm{H}_{4} \mathrm{~N}_{3} \mathrm{O}_{4}+\mathrm{C}_{4} \mathrm{H}_{4} \mathrm{~N}_{3} \mathrm{O}_{3} \mathrm{~K} \text {. }
$$

Trägt man in kochende Kalilauge, die nicht mehr als $20 \mathrm{~g}$ Aetzkali im Liter enthalten soll, rasch trocknes, feinst gepulvertes Uramil ein und filtrirt, wenn die Lösung nur mehr träge erfolgt, rasch auf der Saugpumpe ab, so krystallisiren bei sorgfältigem Luftabschlusse im Verlaufe einiger Stunden feine, durchsichtige, viereckige Blättchen des Kaliumsalzes aus. Bei raschem Arbeiten sind dieselben fast völlig farblos, bei langsamerem färbt sich die Flüssigkeit dunkelroth und die Krystalle sind dann rosa gefärbt. In trocknem Zustande färbt sich das Salz nicht so rasch roth.

I. $0,1581 \mathrm{~g}$, bei $115^{\circ}$ ohne Verlust getrocknet, gaben $0,1717 \mathrm{CO}_{2}$ und $0,0445 \mathrm{H}_{2} \mathrm{O}$. $0,1499 \mathrm{~g}$ gaben $0,0397 \mathrm{~K}_{2} \mathrm{SO} 0$.

$0,1454 \mathrm{~g} \quad " 34,1 \mathrm{ccm}$ Stickgas bei $14^{0}$ und $716,5 \mathrm{~mm}$ Druck.

II. $\mathrm{C}, 1602 \mathrm{~g} \quad " \quad 0,1719 \mathrm{CO}_{2}$.

$0,1918 \mathrm{~g} \quad, \quad 0,0478 \mathrm{~K}_{2} \mathrm{SO}_{1}$. 
Berechnet für

\begin{tabular}{lcrc}
$\mathrm{C}_{4} \mathrm{H}_{5} \mathrm{~N}_{3} \mathrm{O}_{3}+\mathrm{C}_{4} \mathrm{H}_{4} \mathrm{~N}_{3} \mathrm{O}_{3} \mathrm{~K}$ & \multicolumn{1}{c}{ I. } & II. \\
$\mathrm{C}$ & 29,72 & 29,62 & 29,26 \\
$\mathrm{H}$ & 2,78 & 3,15 & - \\
$\mathrm{N}$ & 26,00 & 26,30 & - \\
$\mathrm{K}$ & 12,07 & 11,89 & 12,15
\end{tabular}

Das Salz ist in Wasser kaum leichter löslich als Uramil und lässt sich nicht umkrystallisiren. Bei verschiedenen Versuchen, dasselbe mit Jodmethyl oder Dimethylsulfat trocken zu methyliren, wurde niemals ein dem Kaliumsalz entsprechendes Monomethylderivat erhalten, dagegen neben unverändertem Uramil wenig 1,3-Dimethyluramil.

Darstellung, Aussehen und Verhalten des Natriumsalzes sind vollständig analog.

$0,1334 \mathrm{~g}$ gaben $0,0312 \mathrm{Na}_{2} \mathrm{SO}_{4}$.

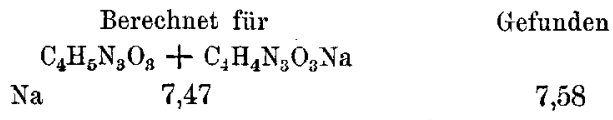

Hauptsächlich durch seine Schwerlöslichkeit stellt sich das Uramil an die Seite derjenigen Verbindungen der Harnsäuregruppe, welche einen complicirteren Bau des Moleküls als das Alloxan besitzen, wie z. B. die Harnsäure selbst und das Alloxantin. Die eben beschriebenen Salze des Uramils, in denen auf zwei Mol. Uramil ein Aequivalent Base trifft, scheinen nun in der That zu beweisen, dass auch das Uramil dazu neigt, ein Doppelmolekül zu bilden. Wäre nun das Uramil wirklich complicirter zusammengesetzt, so müsste aus diesen Salzen ein entsprechend methylirtes Uramil zu gewinnen sein und dessen Existenz wäre für eine complicirte Natur beweisend. Dies ist aber nicht der Fall. Unter dem Einflusse von Alkali treten offenbar zwei Mol. Uramil zu einer lockeren Verbindung zusammen, ähnlich wie bei dem von Baeyer beschriebenen ${ }^{5}$ ) Violantin, das Additionsproduct ist aber zu selbstständigen Reactionen nicht befähigt.

5) Diese Annalen 127, 223. 
Aufspaltung des Vramils.

Lässt man auf Uramil oder seine Alkalisalze mässig concentrirte Kali- oder Natronlauge bei Siedehitze längere Zeit einwirken, so wird der Ring gespalten:

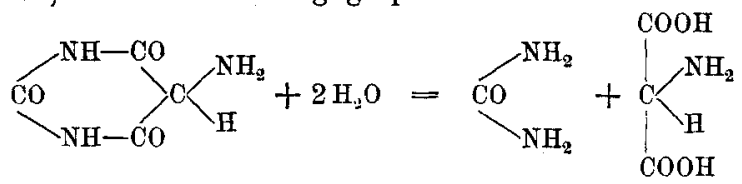

Es entsteht in erster Linie Harnstoff und Amidomalonsäure. Die Reaction verläuft also ganz analog der Spaltung von Violursäure, Alloxan und Barbitursäure in Harnstoff und die entsprechenden zweibasischen Säuren und giebt den directen Beweis für die Constitution des Uramils, Dabei ist sehr auffällig, dass die Amidogruppe gar nicht angegriffen wird. Es konnten in keinem Falle - entgegen der Angabe Liebig und Wöhler's (loc. cit.) - Dialursäure oder irgend welche Umwandlungsproducte derselben oder des Alloxans nachgewiesen werden. Die von Baeyer ${ }^{6}$ ) früher ausgesprochene Vermuthung, dass hier wahrscheinlich die geringere Beständigkeit der Malonsäurederivate eine Spaltung verhindert, kann demnach nicht mehr aufrecht erhalten werden. - Die Amidomalonsäure wurde durch Ueberführung in Glycocoll identificirt.

Neben der Spaltung in Harnstoff und Amidomalonsäure läuft eine zweite Reaction parallel und gestaltet die Einwirkung von Alkali auf Uramil complicirter, als dieselbe z. B. beim Alloxan verläuft. Ein Theil des abgespaltenen Harnstoffs reagirt nämlich weiterhin mit unverändertem Uramil nach folgender Gleichung:

$\overbrace{\mathrm{NH}-\mathrm{CO}}^{\mathrm{NH}-\mathrm{CO}}>_{\mathrm{H}}^{\mathrm{NH}_{2}}+\mathrm{NH}_{2} \cdot \mathrm{CO} \cdot \mathrm{NH}_{2}=\underbrace{\mathrm{CO}}_{\mathrm{NH}-\mathrm{CO}}>_{\mathrm{H}}^{\mathrm{NH}-\mathrm{CO}}\left\langle_{\mathrm{NH} \cdot \mathrm{CO} \cdot \mathrm{NH}_{2}+\mathrm{NH}_{3}}^{\mathrm{No}}\right.$

Die derart gebildete Pseudoharnsäure wurde gleichfalls isolirt und durch ihre charakteristischen Eigenschaften als solche sichergestellt.

6) Diese Annalen 131, 301. 
Endlich spaltet aber das Alkali die gebildete Pseudoharnsäure selbst in Harnstoff und eine bisher unbekannte Säure, die als Carbamidomalonsäure zu bezeichnen ist:

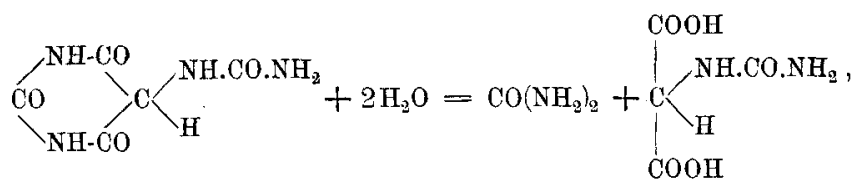

so dass neben einander Amidomalonsäure, Pseudoharnsäure und Carbamidomalonsäure entstehen. Diese letztere lässt sich direct durch anhaltendes Kochen einer alkalischen Lösung von Pseudoharnsäure gewinnen. Ihre Constitution wurde durch Ueberführung in Hydantoïnsäure,

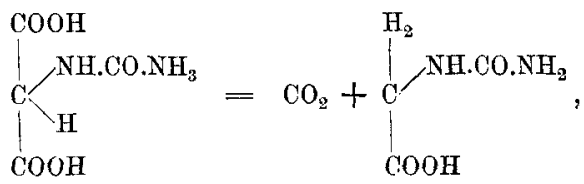

eindeutig festgelegt.

In einem Kolben aus Jenenser Glas mit Rückflusskühler kocht man Uramil mit der zehnfachen Menge zehnprocentiger Kalilauge, bis eine Probe der klaren Lösung beim Versetzen mit Essigsäure nur mehr wenig Flocken von unangegriffenem Uramil absetzt; bei Anwendung von $50 \mathrm{~g}$ Uramil ist dies nach etwa zehn Stunden der Fall. Man lässt erkalten, wobei sich meist schon ein Theil des pseudoharnsauren Kaliums abscheidet, und neutralisirt mit Essigsäure, wodurch sich noch unverändertes Uramil in Flocken abscheidet. Engt man das Filtrat im Wasserbade auf etwa das halbe Volumen ein, so krystallisirt beim Stehen in der Kälte der Rest des pseudoharnsauren Kaliums. Dasselbe wurde aus Wasser (I einmal, II dreimal) umkrystallisirt, wodurch es aber doch nicht rein weiss erhalten werden kounte. 
I. $0,1843 \mathrm{~g}$ gaben $0,1548 \mathrm{CO}_{2}$ und $0,0552 \mathrm{H}_{2} \mathrm{O}$.

$0,1122 \mathrm{~g}, 22,4 \mathrm{ccm}$ Stickgas bei $17^{\circ}$ und $713 \mathrm{~mm}$ Druck.

II. $0,1311 \mathrm{~g} \quad, \quad 0,1105 \mathrm{CO}_{2}$ und $0,0405 \mathrm{H}_{2} \mathrm{O}$. $0,1516 \mathrm{~g}, "$ nach $\mathrm{Kjeldahl} 0,0336 \mathrm{~N}$. $0,2408 \mathrm{~g}$ "verloren bei $160^{\circ} 0,0370 \mathrm{H}_{2} \mathrm{O}$ und gaben 0,0817 $\mathrm{K}_{2} \mathrm{SO}_{4}$.

$\begin{array}{lc} & \text { Berechnet für } \\ & \mathrm{C}_{3} \mathrm{H}_{5} \mathrm{~N}_{4} \mathrm{O}_{4} \mathrm{~K}, 2 \mathrm{H}_{2} \mathrm{O} \\ \mathrm{C} & 23,08 \\ \mathrm{H} & 3,46 \\ \mathrm{~N} & 21,54 \\ \mathrm{~K} & 15,00 \\ \mathrm{H}_{2} \mathrm{O} & 13,85\end{array}$

\begin{tabular}{rr} 
I. & \multicolumn{1}{c}{ II. } \\
22,91 & 22,99 \\
3,35 & 3,45 \\
22,05 & 22,16 \\
- & 15,21 \\
- & 15,36
\end{tabular}

Aus der warmen Lösung des Kaliumsalzes wurde mit Salzsäure die Pseudoharnsäure freigemacht. Sie krystallisirte in kleinen, glänzenden Prismen und zeigte die von Baeyer ${ }^{7}$ ) beschriebenen Eigenschaften.

$0,1945 \mathrm{~g}$, bei $110^{\circ}$ getrocknet, gaben $0,2254 \mathrm{CO}_{2}$ und $0,0668 \mathrm{H}_{2} \mathrm{O}$. $0,1221 \mathrm{~g}$ gaben $32,6 \mathrm{ccm}$ Stickgas bei $17^{\circ}$ und $717 \mathrm{~mm}$ Druck.

$\begin{array}{ccr} & \text { Berechnet für } & \text { Gefunden } \\ & \mathrm{C}_{5} \mathrm{H}_{8} \mathrm{~N}_{4} \mathrm{O}_{4} & \\ \mathrm{C} & 32,25 & 31,61 \\ \mathrm{H} & 3,23 & 3,84 \\ \mathrm{~N} & 30,11 & 29,62\end{array}$

Das Filtrat vom pseudoharnsauren Kalium enthält Amidomalonsäure und Carbamidomalonsäure als Kaliumsalze. Die Trennung beider Säuren gelingt auf folgendem Wege. Man engt auf dem Wasserbade stark ein und fällt vorsichtig mit Alkohol. Die Kalisaize fallen ölig, sehr bald beginnt jedoch die Krystallisation des amidomalonsauren Kaliums und ist in zwei bis drei Tagen vollendet. Das carbamidomalonsaure Kalium bleibt unter diesen Umständen stets ölig und kann leicht getrennt werden. Die weitere Behandlung des amidomalonsauren Kaliums erfolgt nach der von Baeyer ${ }^{8}$ ) gegebenen Vorschrift, indem man es mittelst Bleiacetat in das schwerlösliche Bleisalz verwandelt und

7) Diese Annalen 127, 3.

$\left.{ }^{8}\right)$ Diese Annalen 131, 295. 
aus diesem durch Schwefelwasserstoff die Amidomalonsäure freimacht. Die Isolirung der Carbamidomalonsäure erfolgt ganz analog.

Das durch Alkohol gefällte amidomalonsaure Kalium wurde aus heissem Wasser unter Zusatz von Alkohol umkrystallisirt und bei $115^{0}$ ohne Gewichtsverlust getrocknet.

I. $0,2098 \mathrm{~g}$ gaben $0,1771 \quad \mathrm{CO}_{2}$ und $0,0571 \mathrm{H}_{8} \mathrm{O}$.

$0,1600 \mathrm{~g}, 15,5 \mathrm{ecm}$ Stickgas bei $20,5^{\circ}$ und $715 \mathrm{~mm}$ Druck. $0,1692 \mathrm{~g} \quad, \quad 0,0906 \mathrm{~K}_{2} \mathrm{SO}_{4}$.

II. $0,1802 \mathrm{~g}, \quad$ nach $\mathrm{Kj} \theta 1 \mathrm{dahl} 0,0185 \mathrm{~N}$. $0,1615 \mathrm{~g} \quad, \quad 0,0885 \mathrm{~K}_{2} \mathrm{SO}_{4}$.

\begin{tabular}{lcrl} 
& Berechnet für & \multicolumn{2}{c}{ Gefunden } \\
$\mathrm{C}$ & $\mathrm{C}_{3} \mathrm{H}_{4} \mathrm{NO}_{4} \mathrm{~K}$ & $\mathrm{I}$. & $\mathrm{II}$. \\
$\mathrm{H}$ & 22,93 & 23,02 & - \\
$\mathrm{N}$ & 2,55 & 3,04 & - \\
$\mathrm{K}$ & 8,99 & 10,62 & 10,26 \\
& 24,84 & 24,05 & 24,58
\end{tabular}

Die daraus gewonnene Säure krystallisirte in ziemlich grossen, undeutlich ausgebildeten Prismen; sie wurde über Schwefelsäure bis zur Gewichtsconstanz getrocknet.

$0,1102 \mathrm{~g}$ gaben $13,0 \mathrm{ccm}$ Stickgas bei $23^{\circ}$ und $718 \mathrm{~mm}$ Druck.

$0,1367 \mathrm{~g} \quad, \quad 0,1517 \mathrm{CO}_{2}$ und $0,0668 \mathrm{H}_{2} \mathrm{O}$.

Berechnet für $\mathrm{C}_{3} \mathrm{H}_{3} \mathrm{NO}_{4}$

$\begin{array}{lrr}\mathrm{C} & 30,25 & 30,27 \\ \mathrm{H} & 4,20 & 5,46 \\ \mathrm{~N} & 11,76 & 12,85\end{array}$

Die Differenz im Stickstoff- und Wasserstoffgehalt ist wahrscheinlich auf theilweise Bildung ron Glycocoll zurückzuführen, wofür sich 22,00 pC. C, 6,66 pC. H und 18,66 pC. N berechnen.

Beim Erhitzen hinterliess die Säure einen festen Rückstand, der durch den Schmelzp. $236^{\circ}$ und durch die charakteristischen Reactionen mit dem Glycocoll identificirt wurde.

\section{Carbamidomalonsäure.}

Wie oben geschildert, entsteht diese Säure bei der Spaltung von Uramil durch Alkalien als secundäres Product. Hat die- 
selbe die ihr oben zugeschriebene Constitution, so muss sie auch direct durch Spaltung der Pseudoharnsäure entstehen. In der That lässt sie sich auch aus dieser Säure ohne Schwierigkeit gewinnen. $\mathrm{Zu}$ diesem $\mathrm{Zwecke}$ kocht man reines pseudoharnsaures Kalium mit dem zehnfachen Gewicht zehnprocentiger Kalilauge, bis beim Ansäuern einer Probe mit Salzsäure nach einigem Stehen keine Ausscheidung von Pseudoharnsäure mehr erfolgt, neutralisirt mit Essigsäure und gewinnt wie oben über das Bleisalz die freie Säure.

Die Carbamidomalonsäure krystallisirt in glänzenden, oft charakteristisch eingekerbten Prismen; die erste Krystallisation aus dem Bleisalz dauert oft mehrere Tage. Zum Umkrystallisiren wird sie am besten in Wasser von $30^{\circ}$ gelöst und mit Alkohol gefällt. Sie entbält kein Krystallwasser.

$0,1489 \mathrm{~g}$ gaben $0,1592 \quad \mathrm{CO}_{2}$ und $0,0572 \mathrm{H}_{2} \mathrm{O}$.

$0,1043 \mathrm{~g} \quad, \quad 17 \mathrm{~cm}$ Stickgas bei $21^{\circ}$ und $717 \mathrm{~mm}$ Druck.

Berechnet fïr $\mathrm{C}_{4} \mathrm{H}_{6} \mathrm{~N}_{2} \mathrm{O}_{5}$

$\mathrm{C}$

H

$\mathrm{N}$
29,63

3,70

17,28
Gefunden

29,16

17,85

In kaltem Wasser ist sie schwer, in heissem leicht löslich; bei sehr raschem Arbeiten kann sie daraus umkrystallisirt werden, doch verliert sie dabei leicht Kohlensäure (vergl. unten). In kaltem Alkohol ist sie merklich löslich; Aether fällt sie daraus in ziemlich derben Nadeln. Sie schmilzt bei raschem Erhitzen bei $148--150^{\circ}$ unter Zersetzung.

Die Carbamidomalonsäure ist eine kräftige $\mathrm{zweibasische}$ Säure, die Carbonate, auch Baryumcarbonat, zerlegt. Ihre Schwermetallsalze krystallisiren theilweise sehr schön, weniger schön die Alkalisalze.

Das Kaliumsalz ist in Wasser sehr leicht löslich; beim Fällen mit Alkohol bleibt es stets ölig; aus reiner Säure mit Kaliumcarbonat bereitet, hinterbleibt es beim Verdunsten seiner Lösung über Schwefelsäure undeutlich krystallisirt. 
Das neutrale Ammoniumsalz, aus der Säure mittelst Ammoniak gewonnen, ist in Wasser gleichfalls sehr leicht löslich. Alkohol fällt es aus der wässrigen, auch stark verdünnten Lösung in dünnen, langen, oft lanzettförmig verbreiterten Nadeln ron starkem Glasglanz.

$0,1706 \mathrm{~g}$ verloren im Vacuum bei $70^{\circ} 0,0140 \mathrm{~g}$.

$0,1261 \mathrm{~g}$ gaben $30,3 \mathrm{cem}$ Stickgas bei $24^{\circ}$ und $716 \mathrm{~mm}$ Druck.

Berechnet für

$\begin{array}{lc} & \mathrm{C}_{4} \mathrm{H}_{4} \mathrm{~N}_{2} \mathrm{O}_{5}\left(\mathrm{NH}_{4}\right)_{2} \cdot \mathrm{H}_{2} \mathrm{O} \\ \mathrm{N} & 26,18 \\ \mathrm{H}_{2} \mathrm{O} & 8,41\end{array}$

Gefunden

8,21

Das neutrale Baryumsalz entsteht aus der freien Säure mit Barytwasser oder aus den Salzen mit Chlorbaryum. Es krystallisirt in schönen glasglänzenden Prismen, die in heissem und kaltem Wasser schwer löslich sind.

$0,2522 \mathrm{~g}$ verloren bei $140^{\circ} 0,0274 \mathrm{H}_{2} \mathrm{O}$ und gaben $0,1773 \mathrm{BaSO}_{4}$.

Bereclunet für $\mathrm{C}_{4} \mathrm{H}_{4} \mathrm{~N}_{2} \mathrm{O}_{5} \mathrm{Ba}, 2 \mathrm{H}_{2} \mathrm{O}$

C $\quad 41,14$

$2 \mathrm{H}_{2} \mathrm{O} \quad 10,81$
Gefunden

41,38

10,86

Das Silbersalz entsteht aus dem Ammoniumsalz mittelst Silbernitrat und ist ein flockiger, in kaltem Wasser schwer löslicher Niederschlag, der bald in dicken, zugespitzten Prismen krystallisirt. In wässriger Lösung wird es beim Erwärmen rasch geschwärzt.

Das neutrale Bleisalz, aus dem Kaliumsalz mittelst Bleiacetat dargestellt, fällt zuerst amorph, wird aber bald krystallinisch. Namentlich aus den Mutterlaugen krystallisirt es in schönen, dicken, glasglänzenden Prismen. Es ist in kaltem Wasser schwer, in heissem leichter löslich.

$0,4753 \mathrm{~g}$ verloren bei $140^{\circ} 0,0257 \mathrm{H}_{2} \mathrm{O}$ und gabeu $0,3624 \mathrm{PbSO}_{4}$.

$\begin{array}{lcr} & \text { Berechnet fïr } & \text { Gefunden } \\ & \mathrm{C}_{4} \mathrm{H}_{4} \mathrm{~N}_{2} \mathrm{O}_{5} \mathrm{~Pb}, \mathrm{H}_{2} \mathrm{O} & \\ \mathrm{Pb} & 53,70 & 52,07 \\ \mathrm{H}_{2} \mathrm{O} & 4,67 & 5,41\end{array}$

Beim Kochen einer wässrigen Lösung von Carbamidomalonsüure entweicht Kohlensäure. Engt man nach Reendigung der 
Gasentwickelung im Vacuum ein, so krystallisiren grosse, glasglänzende Prismen von Hydantoïnsäure, die bei $155^{\circ}$ unter Zersetzung schmelzen.

$0,1204 \mathrm{~g}$ gaben $0,1347 \mathrm{CO}_{2}$ und $0,0606 \mathrm{H}_{2} \mathrm{O}$.

$0,1396 \mathrm{~g}, \quad, 31,7 \mathrm{ccm}$ Stickgas bei $22^{\prime \prime}$ und $718 \mathrm{~mm}$ Druek. Berechnet fiir Gefunden $\mathrm{C}_{3} \mathrm{H}_{6} \mathrm{~N}_{9} \mathrm{O}_{3}$

$\begin{array}{lrr}\mathrm{C} & 30,51 & 30,51 \\ \mathrm{H} & 5,08 & 5,63 \\ \mathrm{~N} & 23,73 & 24,58\end{array}$

Erhitzt man Carbamidomalonsäure mit Wasser im Einschmelzrohre auf $140^{\circ}$, so wird als einziges krystallisirtes Product oxalsaures Ammoniak gebildet.

\section{Uramilsäure.}

Liebig und Wöhler bezeichnen eines der Zersetzungsproducte des Uramils durch verdüunte Schwefelsäure als Uramilsäure ${ }^{9}$ ). Die Analyse dieser Verbindung führte sie zu der Formel (in alten Aequivalenten)

$$
\mathrm{C}_{18} \mathrm{H}_{20} \mathrm{~N}_{10} \mathrm{O}_{15}
$$

wobei im Wesentlichen aus zwei Mol. Uramil ein Mol. Ammoniak ausgetreten sein sollte. Da es nicht ausgeschlossen erschien, dass die Uramilsäure mit dem Murexid in einem engen $\mathrm{Zu}$ sammenhange stehe, wurde sie im Anschluss an das Uramil etwas näber untersucht.

Die Vorschrift von Liebig und Wöhler zur Darstellung der Uramilsäure gestattet bei ihrer Unbestimmtheit keine sichere Géwinnung dieser Verbindung. Vielfache eigene Versuche haben gelehrt, dass eine bestimmte Vorschrift auch nicht gegeben werden kann, da die Bildung zu unübersichtlich verläuft. Am besten gelang dieselbe nach folgender Methode.

$2,5 \mathrm{~g}$ thionursaures Ammoniak wurden in Wasser von $60^{\circ}$ gelöst, mit 20 Tropfen verdünnter Schwefelsäure versetzt und bei dieser Temperatur zur Trockne verdampft. Lösen und

9) Diese Annalen 26, 314 . 
Abdampfen bei dieser Temperatur wurde - unter Zusatz von zwei Tropfen verdünnter Schwefelsäure etwa bei jedem zweiten Abdampfen - so oft wiederholt, bis der anfangs rothe, schön krystallisirte Abdampfungsrückstand dauernd gelb und undeutlich krystallisirt war. Die gelblichen Krystalle wurden nach mehrmaligem Umkrystallisiren aus Wasser ziemlich farblos, jedoch konnten sie auch bei Anwendung von Thierkohle nicht rein weiss erhalten werden. Neben diesen Krystallen tritt stets unverändertes thionursaures Salz und Uramil, zuweilen auch Alloxantin auf.

$0,1762 \mathrm{~g}$, exsiccatortrocken, verloren bei $110^{\circ}$ nichts an Gewicht und gaben $0,2267 \mathrm{CO}_{2}$ und $0,0534 \mathrm{H}_{2} \mathrm{O}$.

$0,1258 \mathrm{~g}$ gaben $28,9 \mathrm{ccm}$ Stickgas bei $15,5^{n}$ und $723 \mathrm{~mm}$ Druck.

$\begin{array}{ccr} & \text { Berechnet für } & \text { Gefunden } \\ & \mathrm{C}_{8} \mathrm{H}_{9} \mathrm{~N}_{5} \mathrm{O}_{0} & \\ \mathrm{C} & 35,42 & 34,95 \\ \mathrm{H} & 3,32 & 3,38 \\ \mathrm{~N} & 25,83 & 25,82\end{array}$

Es gelang nicht, die Uramilsäure in ,ziemlich starken" (nach Liebig und Wöhler!) Prismen zu gewinnen. Die Krystalle blieben stets kleinprismatisch, zeigten aber starken Glasglanz. Alle übrigen Eigenschaften stimmen mit der Beschreibung von Liebig und Wöhler völlig überein, insbesondere die nur schwach saure Reaction und das Verhalten gegen concentrirte Salpetersäure.

Mit Calciumbydroxyd entwickelt die Uramilsäure in der Kälte reichlich Ammoniak; sie ist also gar keine Säure, sondern ein Ammoniumsalz. Dasselbe entsteht bei Gegenwart von freier Schwefelsäure; das Salz wird also von Mineralsäuren nicht zerlegt. Dies ist aber ganz das Verhalten einer schon bekannten Säure der Harnsäuregruppe, der vou Scblie per ${ }^{10}$ ) entdeckten, von $\mathrm{Ba} \in \mathrm{y} e \mathrm{r}^{11}$ ) eingehend untersuchten Hydurilsäure.

Uramilsäure ist nun in der That nichts anderes als saures lydurilsaures Ammoniak. Sie giebt in genügend reinem $\mathrm{Zu}$ -

10) Diese Annalen 56, 11.

11) Diese Annalen 132, 302. 
stande die für Hydurilsäure charakteristische Grünfärbung mit Eisenchlorid und die oben aus den Analysenzahlen berechnete Formel ist die des sauren hydurilsauren Ammoniaks, $\mathrm{C}_{8} \mathrm{H}_{9} \mathrm{~N}_{5} \mathrm{O}_{6}$.

Dass die von Liebig und Wöhler gegebenen Analysenzahlen davon etwas abweichen, hat sehr wahrscheinlich seinen Grund in einer Beimengung freier Hyduriisäure $(+2$ aq.) in ihren Präparaten, wodurch die Procentzahlen für Kohlenstoff und Stickstoff etwas gedrückt werden.

\section{7-Acetyluramil.}

Kocht man zwei Theile feingepulvertes Uramil, einen Theil geschmolzenes Natriumacetat und 15 Theile Essigsäureanbydrid unter Rückfluss etwa eine halbe Stunde, so wird das Uramil acetylirt; dem entstehenden Producte kommt die Formel

Zl1.

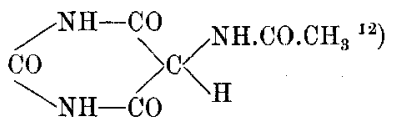

Ein grosser Ueberschuss von Anhydrid ist nöthig, um ein Zusammenbacken und Verkohlen des Uramils zu verhindern. Zur Reinigung wird das Acetylproduct mit gewöhnlichem Ammoniak behandelt, bis der Geruch nicht mehr verschwindet, und das gebildete Ammoniumsalz aus heissem Wasser umkrystallisirt. Säuert man dann eine concentrirte kalte Lösung des Ammoniumsalzes mit Salzsäure an, so krystallisirt das Acetylproduct in concentrisch angeordneten, glasglänzenden Prismen, die sehr oft leicht gelblich gefärbt sind; sie enthalten kein Krystallwasser.

$0,1921 \mathrm{~g}$ gaben $0,2706 \mathrm{CO}_{2}$ und $0,0701 \mathrm{H}_{2} \mathrm{O}$.

$0,110 ̄ 7 \mathrm{~g} " 23,4 \mathrm{~cm}$ Stickgas bei $18^{\circ}$ und $723 \mathrm{~mm}$ Druck. Berechnet für Gefunden $\mathrm{C}_{6} \mathrm{H}_{7} \mathrm{~N}_{3} \mathrm{O}_{4}$

$\mathrm{C}$ 38,92 38,42

$\mathrm{H} \quad 3,78$ 4,08 $\mathrm{N}$ 22,70 22,57

12) Diese Annalen 251, 240. 
Das Acetyluramil reagirt sauer; es macht aus den Alkalicarbonaten Kohlensäure frei. In kaltem Wasser ist es sehr schwer, in heissem leichter löslich. Beim Kochen mit Wasser wird es theilweise verseift, so dass es aus Wasser nicht gut umkrystallisirt wèrden kann. Rascher erfolgt die Verseifung beim Kochen mit verdünnten Mineralsäuren unter Abscheidung der flimmernden Blättchen des Uramils. Beim Erwärmen reducirt es ammoniakalische Silberlösung.

Das Acetyluramil bildet sehr schön krystallisirende Salze. Die analysirten, durch Neutralisiren mit Kalilauge und Ammoniak dargestellten sind einbasisch. Da sie sich jedoch im Ueberschusse der angewandten Base leicht lösen, so ist es möglich, dass auch zweibasische Salze existiren. Aus den Alkalisalzen werden die Schwermetallsalze durch doppelte Umsetzung gewonnen. Die Alkalisalze sind schon in kaltem Wasser ziemlich leicht, in heissem leicht löslich, die übrigen in kaltem Wasser schwer, in heissem ziemlich schwer löslich.

Das Kaliumsalz bildet glasglänzende, anscheinend rhombische Prismen; es krystallisirt ohne Krystallwasser.

$0,1601 \mathrm{~g}$ gaben $0,0614 \mathrm{~K}_{2} \mathrm{SO}_{4}$.

$\begin{array}{ccc} & \text { Berechnet fiur } & \text { Gefunden } \\ & \mathrm{C}_{6} \mathrm{H}_{6} \mathrm{~N}_{3} \mathrm{O}_{4} \mathrm{~K} & \\ \mathrm{~K} & 17,49 & 17,21\end{array}$

Das Ammoniumsalz krystallisirt in feinen, verfilzten Nadeln. Sie haben nicht selten eine schwach gelbliche Farbe, da die Lösung des Salzes sich beim Kochen leicht röthlich färbt. Das Salz färbt sich in feuchtem Zustande an der Luft rosa; es enthält gleichfalls kein Krystallwasser.

$0,1095 \mathrm{~g}$ gaben $27,9 \mathrm{ccm}$ Stickgas bei $15^{\circ}$ und $707 \mathrm{~mm}$ Druck.

$\begin{array}{ccc} & \text { Berechnet für } & \text { Gefunden } \\ & \mathrm{C}_{6} \mathrm{H}_{10} \mathrm{~N}_{4} \mathrm{O}_{4} & \\ \mathrm{~N} & 27,72 & 28,10\end{array}$

Das Silbersalz bildet feine, weisse, zu Warzen vereinigte Nädelchen, die sich beim Kochen in der Flüssigkeit schwärzen. 
Das Baryt- und das Bleisalz bilden feine, weisse Nadeln, die zu charakteristisch nierenförmigen Aggregaten vereinigt sind.

Das Kupfersalz bildet gelbgrüne, rhombische Täfelchen.

\section{Oxydation von Uramil mittelst Kaliumpermanganat.}

Wie das Verhalten des Uramils gegen Alkalien sehr verschiedenartig ist je nach den Versuchsbedingungen, so mannigfaltig sind auch die Producte der Oxydation je nach Wahl des Oxydationsmittels.

Verdünnte Salpetersäure greift, wie Liebig und Wöhler ${ }^{13}$ ) gefunden, lebhaft an und bildet Alloxan. Einwirkung von Silberoxyd oder Quecksilberoxyd, von Luftsauerstoff oder unterchloriger Säure bei Gegenwart von Alkali führen zum Murexid. Auch Jod oxydirt eine alkalische Lösung von Uramil. Während aber anch hier reichliche Mengen von Murexidkalium isolirt werden können, wird unter sonst gleichen Verhältnissen aus Uramil durch Kaliumpermanganat keine Spur Murexid gebildet, obwohl das Oxydationsmittel sofort reducirt wird. Die Oxydation verläuft in ganz anderem Sinne.

Man übergiesst reines, namentlich schwefelsäurefreies Uramil mit etwa dem anderthalbfachen Gewicht 50 procentiger Kalilauge, so dass ein ganz dicker Brei entsteht und lässt einige Zeit stehen, wodurch die ganze Masse zu Dikaliumuramil erstarrt. Dieses löst sich nun leicht in relativ wenig kaltem Wasser. Nan oxydirt man unter Kühlung mit Eiswasser durch vorsichtiges Zufliessenlassen einer concentrirten Kaliumpermanganatlösung, bis in einer Tüpfelprobe auf Fliesspapier eine Grünfärbung die Gegenwart von Manganat anzeigt. Dieses zerstört man eventuell durch wenig Alkohol und filtrirt vom Braunstein ab. Das Filtrat wird mit Essigsäure versetzt, so dass es eben noch alkalisch reagirt, und im Vacuum auf ein kleines Volumen eingeengt. Auf vorsichtigen Zusatz von Methylalkohol wird ein öliges Kalisalz gefällt, das nach einiger Zeit in haken-

13) Diese Annalen 26, 275 und 314. 
artig gebogenen Krystallen anschiesst. Ein grosser Theil der Fällung - namentlich auf Zusatz von mehr Alkohol - bleibt jedoch auch nach längerem Stehen ölig und ist durch keinerlei Reinigung zum Krystallisiren zu bringen; auch andere krystallisirte Salze, z. B. Barytsalze, lassen sich nicht daraus gewinnen.

Die Krystalle werden auf Thon vom anhaftenden Syrup befreit, in kaltem Wasser gelöst und durch vorsichtigen Zusatz von Methylalkohol wieder ausgefällt. Auch bei dieser Behandlung bleiben die letzten Fällungen des vorher schon krystallisirten Salzes ölig, was auf eine theilweise Zersetzung desselben zu deuten scheint.

Die Analyse der zweimal aus Wasser und Methylalkohol umkrystallisirten, lufttrockenen Substanz ergab folgende Zahlen:

I. $0,2340 \mathrm{~g}$ verloren bei $110^{\circ} 0,0129 \mathrm{~g}$ und gaben $0,1914 \mathrm{CO}_{2}$ und $0,0461 \mathrm{H}_{2} \mathrm{O}$.

$0,1423 \mathrm{~g}$ gaben $0,0658 \mathrm{~K}_{2} \mathrm{SO}_{4}$.

II. $0,1998 \mathrm{~g}$ verloren bei $110^{\circ} 0,0112 \mathrm{~g}$ und gaben $0,0861 \mathrm{~K}_{2} \mathrm{SO}_{4}$. $0,1849 \mathrm{~g}$ gaben $0,1541 \mathrm{CO}_{2}$ und $0,0465 \mathrm{H}_{2} \mathrm{O}$.

$0,1013 \mathrm{~g}, 17,8 \mathrm{ccm}$ Stickgas bei $15^{\circ}$ und $719 \mathrm{~mm}$ Druck.

Berechnet für $\mathrm{C}_{4} \mathrm{H}_{4} \mathrm{~N}_{3} \mathrm{O}_{4} \mathrm{~K} \cdot{ }_{1} / \mathrm{H}_{2} \mathrm{O}$

$\mathrm{C}$

23,41

$\mathrm{H} \quad 2,44$

$\mathrm{N} \quad 20,48$

I. $\frac{\text { Gefunden }}{\text { II. }}$

K

19,02

$22,31 \quad 22,73$

$2,81 \quad 2,82$

$\mathrm{K}$

4,50

- $\quad 19,70$

$19,70 \quad \mathbf{1 9 , 3 6}$

$5,51 \quad 5,60$

Die zu diesèr Verbindung gehörige freie Säure ist nicht beständig. Säuert man das Kaliumsalz mit Mineralsäuren oder auch mit Essigsäure an, so beginnt schon in der Kälte theilweise Zersetzung unter Koblensäureentwickelung. Erwärmt man die essigsaure Lösung des Kaliumsalzes auf etwa $40^{\circ}$, bis keine Kohlensäure mehr entweicht, so erfolgt auf Zusatz von Methylalkohol die Fällung eines Syrups, der bald grossentheils krystallinisch erstarrt. In Wasser gelöst und mit Methylalkohol wieder ausgefällt, krystallisiren kleine, undeutlich ausgebildete Prismen, aus verdünnter wässriger Lösung mit sehr viel Methyl- 
alkohol undurchsichtige Krystallkugeln: ganz dieselben Krystallisationserscheinungen, die saures alloxansaures Kalium zeigt. Die Analyse bestätigte, dass saures alloxansaures Kalium vorlag.

$0,2492 \mathrm{~g}$, lufttrocken, zeigten bei $115^{n}$ keine Gewichtsabnahme und gaben $0,21.60 \mathrm{CO}_{2}$ und $0,0400 \mathrm{H}_{2} \mathrm{O}$.

$0,1777 \mathrm{~g}$ gaben $0,0808 \mathrm{~K}_{\mathbf{g}} \mathrm{SO}_{\mathbf{1}}$.

$0,1336 \mathrm{~g} \quad$ " $16 \mathrm{~cm}$ Stickgas bei $11,5^{\circ}$ und $729,5 \mathrm{~mm}$ Druck. Berechnet für Gefunden $\mathrm{C}_{4} \mathrm{H}_{3} \mathrm{~N}_{2} \mathrm{O}_{5} \mathrm{~K}$

$\begin{array}{rrr}\mathrm{C} & 24,24 & 23,64 \\ \mathrm{H} & 1,51 & 1,80 \\ \mathrm{~N} & 14,14 & 13,75 \\ \mathrm{~K} & 19,69 & 20,40\end{array}$

Die Entwickelung von Kohlensäure steht mit der Bildung der Alloxansäure offenbar nicht in directem Zusammenhange, da dieselbe die gleiche Anzahl Kohlenstoffatome enthält wie das oben beschriebene Kaliumsalz, sie muss daher aus einer parallel mit der Alloxansäurebildung sich abspielenden Nebenreaction herrühren.

Im Filtrat des mit Methylalkohol gefällten Salzes findet sich reichlich essigsaures Ammoniak; es ist also Ammoniak abgespalten worden.

Ebensowenig beständig wie gegen Säuren ist die Verbindung $\mathrm{C}_{4} \mathrm{H}_{4} \mathrm{~N}_{3} \mathrm{O}_{4} \mathrm{~K}$ gegen Alkalien. Hält man die alkalische Lösung des Salzes längere Zeit bei nur $40^{\circ}$, so wird Ammoniak abgespalten und man erhält neutrales alloxansaures Kalium auf Zusatz von Alkohol in glänzenden, alloxantinähnlichen Prismen.

$0,3141 \mathrm{~g}$, lufttrocken, verloren bei $\left.115^{\circ} 0,0487 \mathrm{H}_{2} \mathrm{O}^{14}\right)$.

$0,13 \mathrm{~s} 4 \mathrm{~g}$, bei $115^{\circ}$ getrocknet, gaben $14,5 \mathrm{ccm}$ Stickgas bei $21^{\circ}$ und $723 \mathrm{~mm}$ Druck.

\begin{tabular}{|c|c|c|}
\hline \multicolumn{3}{|c|}{$\begin{array}{c}\text { Berechnet fïr } \\
\mathrm{C}_{4} \mathrm{E}_{2} \mathrm{~N}_{2} \mathrm{O}_{5} \mathrm{~K}_{2} \cdot 3 \mathrm{H}_{2} \mathrm{O}\end{array}$} \\
\hline $\left.2^{1 / 2} \mathrm{H}_{2} \mathrm{O}^{14}\right)$ & 15,50 & 15,60 \\
\hline $\mathrm{N}$ & 11,42 & 11,82 \\
\hline
\end{tabular}

14) Ueber den Wasserverlust vergl. Schlieper, diese Annalen $\mathbf{5 5}$, 260. Die Angabe Schlieper's, das nentrale alloxansaure Kalium 
Dieses Salz erhält man daher auch, wenn man bei der oben beschriebenen Darstellung nicht mit Essigsäure abstumpft, sondern die alkalische Lösung direct eindampft und dann mit Alkohol fällt.

Weder durch Natriumamalgam noch durch Schwefelammonium oder Schwefelwasserstoff kann die Verbindung wieder zu Uramil reducirt werden. Saure Reductionsmittel verbieten sich wegen der Zersetzlichkeit mit Säuren von selbst.

Das Salz $\mathrm{C}_{4} \mathrm{H}_{4} \mathrm{~N}_{3} \mathrm{O}_{4} \mathrm{~K}$ ist isomer mit dem von Strecker durch alkalische Oxydation von Harnsäure dargestellten, von Medicus ${ }^{15}$ ) beschriebenen oxonsauren Kalium.

Soweit die Angaben über dieses Salz reichen, scheint sich auch sein Verhalten mit dem des Oxydationsproductes aus Uramil zu decken. Doch liefert das oxonsaure Kalium bei der Zersetzung mit Essigsäure unter Abspaltung von Ammoniak und Kohlensäure Kaliumglyoxylharnstoff,

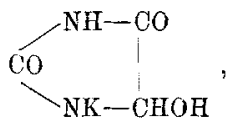

während bei Einwirkung von Essigsäure auf die beschriebene Verbindung alloxansaures Kalium isolirt wurde. Dieser Widerspruch ist jedoch keineswegs unüberwindlich, denn da bei dieser Zersetzung auch Kohlensäureentwickelung beobachtet wurde, so ist es denkbar, dass auch hier eine Nebenreaction - unter Abspaltung von Kohlensäure und Ammoniak - zum Kaliumglyoxylharnstoff fübrt, der sich aber der Isolirung entzog und sich in dem nicht zur Krystallisation zu bringenden Syrup befand. Jedoch war eine genaue kritische Vergleichung mit oxonsaurem Kalium zur Prüfung dieser Annahme nicht möglich, da wir nicht in der Lage waren, den langwierigen Weg zu seiner Darstellung zu wiederholen.

reagire neutral, ist nicht richtig; eine nach seiner Vorschrift dargestellte, sorgfältig gereinigte und schön krystallisirte Probe des Salzes reagirte stark alkalisch.

15) Diese Annalen 175, 232. 
Medicus giebt ${ }^{16}$ ) von der Structur der Oxonsäure folgendes, experimentell nicht bewiesenes Bild:<smiles></smiles>

Dieselbe könnte aber nach ihrer Entstehung aus Harnsäure ebenso gut folgendermassen constituirt sein:

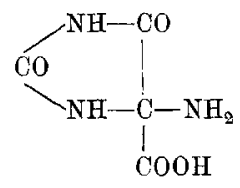

sodass sie als Amidohydantoïncarbonsäure zu bezeichnen wäre. Diese letztere Formel kann aber auch für das Oxydationsproduct aus Uramil als sehr wahrscheinlich betrachtet werden; denn die Verbindung kann nicht mehr zu Uramil reducirt werden, ist also kein Oxyuramil,<smiles>NC(O)(O)C(=O)NCCO</smiles>

(worauf die empirische Zusammensetzung gleichfalls stimmen würde).

Der in der ersten Formulirung anzunebmende Uebergang des Sechsrings in den Fünfring ist durch eine analoge Reaction schon bekannt; Alloxan geht bei der Oxydation mit Salpetersäure in Parabansäure über:

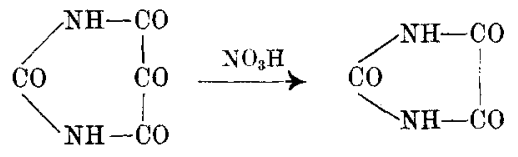

12) Ber. d. deutsch. chem. Ges. 10, 545 . 
Die Zersetzung mittelst Essigsäure wäre dann folgendermassen $z \mathfrak{u}$ erklären:

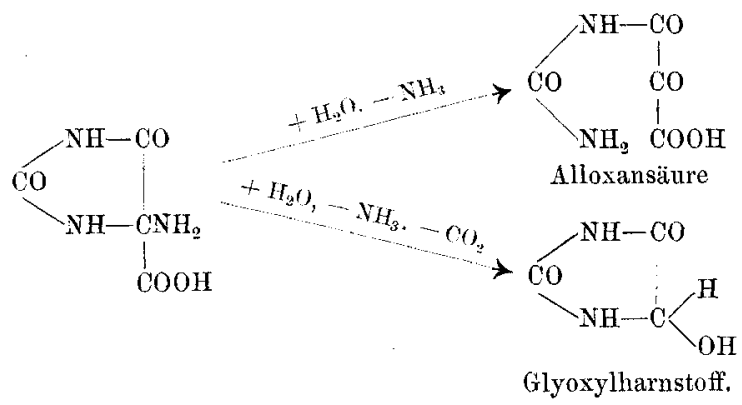

Soweit demnach die Kenntniss der neuen Verbindung reicht, könnte dieselbe als Amidohydantoincarbonsäure bezeichnet werden.

Es war nicht ausgeschlossen, dass durch analoge Durchführung der Oxydation mit einem substituirten Uramil die angegebene Constitutionsformel gestützt werden konnte. $\mathrm{Zu}$ einem solchen Versuche wurde die Pseudoharnsäure gewählt. Verfährt man nun ganz ebenso wie bei der Oxydation des Uramils angegeben, - indem man reines pseudoharnsaures Kalium mit möglichst wenig Kalilauge kalt in Lösung bringt so krystallisirt beim Versetzen mit Alkohol in vahezu quantitativer Ausbeute ein Kaliumsalz in prächtigen Prismen, das sich aber als neutrales alloxansaures Kalium erwies.

$\begin{array}{lcc} & \text { Berechnet } & \text { Gefunden } \\ \mathrm{H}_{2} \mathrm{O} & 15,50 & 16,30 \\ \mathrm{~K} & 26,90 & 26,14\end{array}$

Ein Product, das ähnlich wie beim Uramil noch die unveränderte Anzahl Atome Stickstoff im Molekül enthält, konnte also hier nicht isolirt werden. Schon beim Uramil hat sich ein solches Product als sehr unbeständig gezeigt, hier scheint es in der alkalischen Lösung überhaupt nicht mehr existenzfähig zu sein; im Hinblick auf das Endproduct scheint aber die Reaction dennoch im selben Sinne zu verlaufen. 
Constitution der Thionursäure; Alloxandimethylaminsulfit.

Vielfach variirte Versuche, das 7-Dimethyluramil,<smiles>CCC(C)(C)C(=O)NCCO</smiles>

zu gewinnen, scheiterten an der Unmöglichkeit, die Bildungsweisen des Uramils für die Gewinnung dieser Verbindung entsprechend modificiren zu können. Dagegen haben diese Versuche einen Weg gezeigt, über die Constitution der Thionursäure eine Entscheidung herbeizufübren.

Kocht man Alloxantin mit einer Lösung von Dimethylaminacetat, so bleibt die Lösung klar, beim Einengen scheidet sich kein substituirtes Uramil ab, sondern dialursaures Dimethylamin in glänzenden, quergestreiften Prismen. Versucht man, aus dialursaurem Dimethylamin analog dem früher beschriebenen Versuche durch trocknes Erhitzen Wasser abzuspalten, so verschmiert das Salz unter Braunfärbung schon bei etwa $130^{\circ}$, und es lässt sich daraus nur eine kleine Menge Hydurilsäure isoliren. Die directe Methylirung des Uramils führt zum 1,3Dimethyluramil.

Auch der sonst einfachste Weg, die Abspaltung der Sulfogruppe aus dem entsprechenden thionursauren Salz mittelst Säuren, versagt hier vollständig. E. F ischer hat auf diese Weise (durch Combination von Methylaminsulfit mit den entsprechenden Alloxanen und darauf folgende Spaltung) 7 -monoalkylirte Uramile, z. B. 7-Methyl- ${ }^{17}$ ), 1,7-Dimethyl-18), 1,3,7-Trimethyluramil ${ }^{19}$ ) ohne Schwierigkeit erhalten.

Aus Alloxan und neutralem Dimethylaminsulfit erhält man zwar auch eine sehr schön krystallisirte Verbindung, welche sich in der Zusammensetzung von dem zu erwartenden dimethylthionursauren Dimethylamin nur durch den Mehrgehalt

\footnotetext{
17) Ber. d. dentsch. chem. Ges. B0, 561.

18) Ber. d. deutsch. chem. Ges. B0, 3095.

$\left.{ }^{19}\right)$ Ber. d. deutsch. chem. Ges. 30, 504.
} 
von einem Mol. Wasser unterscheidet. Allein beim Spalten dieser Verbindung wird kein entsprechendes Uramil unter Austritt von Schwefelsäure gebildet, wie dies sonst bei thionursauren Salzen der Fall ist, sondern die schweflige Säure entweicht nahezu quantitativ und Alloxan wird unverändert zurückgewonnen.

Zur Darstellung der Verbindung werden $4 \mathrm{~g} 33$ procentige Dimethylaminlösung unter guter Kühlung mit schwefliger Säure gesättigt und dann mit Dimethylaminlösung neutralisirt. Hierzu giebt man eine concentrirte wässrige Lösung von Alloxan und erwärmt die Mischung etwa $1 \frac{1}{2}$ Stunden auf dem Wasserbade. Man engt dann im Vacuum bei etwa $40^{\circ}$ stark ein, wobei sich die Flüssigkeit allmählich mit einem Brei von haarfeinen, nadeligen und derben prismatischen Krystallen erfüllt.

I. $0,2291 \mathrm{~g}$, exsiccatortrocken, gaben $0,2479 \mathrm{CO}_{2}$ und $0,1455 \mathrm{H}_{2} \mathrm{O}$. $0,1850 \mathrm{~g}$ gaben $29,3 \mathrm{ccm}$ Stickgas bei $14,5^{\circ}$ and $709 \mathrm{~mm}$ Druck. $0,1961 \mathrm{~g}, \quad, \quad 0,1413 \mathrm{BaSO}_{4}$ (nach Carius).

II. $0,1971 \mathrm{~g}$, exsiccatortrocken, gaben $0,2143 \mathrm{CO}_{2}$ und $0,1220 \mathrm{H}_{2} \mathrm{O}$. $0,1286 \mathrm{~g}$ gaben $20,4 \mathrm{~cm}$ Stiekgas bei $14^{0}$ und $711 \mathrm{~mm}$ Druck. $0,1324 \mathrm{~g} \quad, \quad 0,1007 \mathrm{BaSO}_{4}$ (nach Carius).

\begin{tabular}{lcrr} 
& Berechnet fïr & \multicolumn{2}{c}{ Gefunden } \\
& $\mathrm{C}_{8} \mathrm{H}_{18} \mathrm{~N}_{4} \mathrm{O}_{7} \mathrm{~S}$ & \multicolumn{1}{c}{ I. } & II. \\
$\mathrm{C}$ & 30,57 & 29,51 & 29,65 \\
$\mathrm{H}$ & 5,73 & 7,10 & 6,92 \\
$\mathrm{~N}$ & 17,83 & $17,5 \overline{0}$ & 17,66 \\
$\mathrm{~S}$ & 10,19 & 9,89 & 10,44
\end{tabular}

Analyse I ist mit haarfeinen, nadeligen, Analyse II mit prismatischen Krystallen durchgeführt. Sie sind Krystallformen ein und derselben Verbindung. Dieselbe ist in kaltem Wasser spielend löslich, in organischen Lösungsmitteln unlöslich. Sie lässt sich aus sehr verdünntem heissen Alkohol oder durch Lösen in Wasser und Fällen mit Alkohol bequem umkrystallisiren. Meist fallen hierbei zuerst feine Nadeln, dann grosse Prismen.

In trocknem Zustande färbt sich die Verbindung an der Luft schwach rosaroth. Beim Erhitzen auf $120^{\circ}$ tritt Geruch 
nach Dimethylamin auf. Die Verbindung färbt sich dabei prachtvoll tiefroth und nimmt, in der Reibschale verrieben, sehr schönen Murexidglanz an.

Versetzt man eine wässrige Lösung der Verbindung mit verdünnter Salzsäure und erwärmt, so entweicht 'reichlich schweflige Säure. Lässt man erkalten, wenn der Geruch nach schwefliger Säure verschwunden, so krystallisirt nach einigem Stehen etwas Alloxantin, das an der Krystallform und der violetten Fällung mit Barytwasser leicht kenntlich ist. Verdampft man das Filtrat vom Alloxantin im Vacuum zur Trockne, so hinterbleibt eine undeutliche Krystallmasse, aus der mit wenig Alkohol das salzsaure Dimethylamin ausgezogen werden kann. Der Rückstand erweist sich als Alloxan, das an der Blaufärbung mit Ferrosulfat kenntlich ist. Das Alloxantin, das stets nur in kleiner Menge auftritt, verdankt hier seine Entstehung einer reducirenden Wirkung der durch die Salzsäure in Freiheit gesetzten schwefligen Säure. Von einem substituirten Uramil ist keine Spur aufzufinden.

Die wässrige Lösung des Salzes kann einige Zeit gekocht werden, ohne dass Zersetzung eintritt; sie färbt sich dabei hellroth. Um zu versuchen ob eine Umsetzung zu dimethylthionursaurem Salz nicht bei höherer Temperatur zu erzielen ist, wurde cine concentrirte wässrige Lösung des Salzes unter Zusatz von einigen Tropfen Dimethylamin im Einschmelzrohre eine Stunde auf $140^{\circ}$ erhitzt. Beim Oeffnen der Röhre entwich viel Kohlensäure, die Flüssigkeit enthielt nur mehr Schwefelsäure, schweflige Säure, Ammoniak und Dimethylamin, die Substanz war also vollständig zerstört worden.

Die Verbindung von Alloxan mit Dimethylaminsulfit vermag durch doppelte Umsetzung - auch mit Schwermetallsalzen - eine Reihe von Salzen zu bilden, die sämmtlich sehr schön krystallisirt und unbegrenzt beständig sind. Zwei Mol. Dimethylamin sind in ihnen durch zwei Aequivalente einer anderen Base ersetzt; aus allen entweicht beim Ansäuern schweflige Säure. 
So erhält man durch Umsetzen einer nicht zu verdunnten wässrigen Lösung des Salzes mit Chlorkalium das Kaliumsalz in derben Prismen.

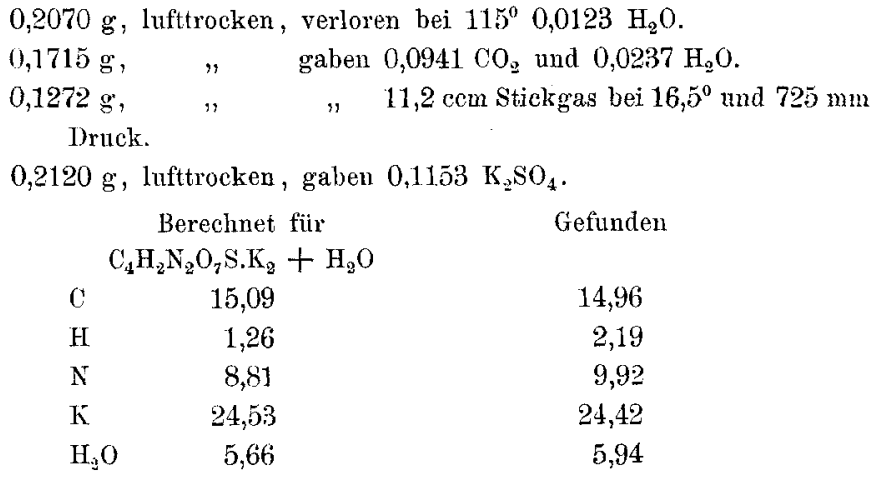

Das Ammoniumsalz bildet rechteckige, in kaltem Wasser schwerlösliche Tafeln, die in Exsiccator getrocknet, wasserfrei sind.

$0,1556 \mathrm{~g}$ gaben $31,5 \mathrm{~cm}$ Stickgas bei $15^{0}$ und $715 \mathrm{~mm}$ Druck.

$\begin{array}{ccc} & \text { Berechnet für } & \text { Gefunden } \\ \mathrm{N} & \mathrm{C}_{4} \mathrm{H}_{10} \mathrm{~N}_{4} \mathrm{O}_{7} \mathrm{~S} & \\ & 21,71 & 22,57\end{array}$

Ėbenso lässt sich mit Chlorbaryum das Baryumsalz als gallertartiger, bald krystallinisch werdender Niederschlag gewinnen. Aus heiss gemischten, sehr verdünnten Lösungen krystallisirt es in breiten, lanzettförmigen Nadeln. Es enthält zwei Mol. Krystallwasser.

Das Bleisalz, mittelst Bleiacetat dargestellt, fällt in der Kälte gleichfalls gelatinös, aus der warmen Lösung krystallisirt es in kleinen warzigen Aggregaten. Es ist in Wasser sehr schwer löslich. Versucht man, selbst unter Eiskühlung dieses Salz mittelst Schwefelwasserstoff zu zersetzen, so tritt sofort Reduction unter Abscheidung von Schwefel ein.

Aus den Ergebnissen der Analyse und nach dem beschriebenen Verhalten lässt sich für die Verbindung aus Alloxan und Dimethylaminsulfit ungezwungen folgende Formel aufstellen: 


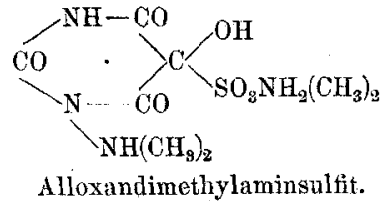

Nach dieser Formel entstehen die anderen Salze durch Austausch des Dimethylamins gegen andere Basen:<smiles>Nn1cnc(=O)c(O)c1</smiles>

und<smiles>O=C1NC=NCC1(O)O</smiles>

Diese Formulirung giebt ein einfaches Bild der Entstehung der Verbindung durch Anlagerung von schwefliger Säure an Alloxan und erklart den leichten Zerfall in die Componenten: Sie schliesst sich an an die für die Bisulfitadditionsproducte der Aldehyde und Ketone gebräuchliche Formel:

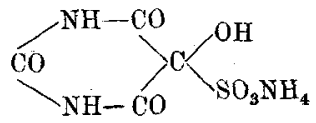

Alloxanammoniumsulfit ${ }^{20}$ ).

Wie aus einem Vergleich beider Formeln hervorgeht, unterscheiden sich die Bisulfitverbindungen nur um den Mindergehalt eines Aequivalents Base von den neuen Körpern. Da sich beide Reiben von Verbindungen auf dieselbe Weise bilden und beim Ansäuern beide ganz gleich in ihre Componenten zerfallen, so ist für beide auch die gleiche Constitution sicher.

In der Verbindung<smiles>CCNCCCN</smiles>

ist es offenbar gelungen, ein Zwischenstadium in der Bildung der Thionursäure aus Alloxan zu fassen, das einen interessanten

20) Bisulfitverbindungen sind speciell von Alloxan in grösserer Zahl bekannt und untersucht (Pellizari, diese Annalen 248, 147). Anxalen der Chemie 333. Bd. 
Schluss auf die Constitution der ersteren gestattet. Es finden sich für ihre Constitution zwei Formeln angegeben:

I.<smiles>CC(N)(O)C(=O)NCCO</smiles>

II.

und<smiles>CCCCNC1NCNC1=O</smiles>

zwischen denen bis jetzt ein experimenteller Entscheid nicht erfolgt ist. Nachdem es aber, wie oben beschrieben, auf keine Weise möglich gewesen ist, bei Anwendung von Dimethylaminsulfit die entsprechend substituirte Thionursäure zu erhalten, sondern die Reaction - die bei Anwendung von Ammoniak und primären Aminen glatt weiter geht - hier bei der Bildung eines einfachen Additionsproductes stehen geblieben ist, so ist hier offenbar die Fortsetzung der Reaction nicht möglich, d. h. die entsprechende Dimethylthionursäure kann sich nicht bilden. Dies ist aber nur bei Annahme der Formel II für die Thionursäure möglich; nach Formel I müssten secundäre Amine im selben Sinne wie primäre reagiren können. Für die Thionursäure ist demnach die Formel

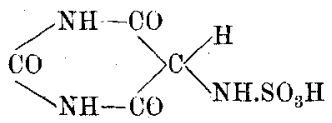

als die richtigere zu bezeichnen.

Umgekehrt ist es bei Annahme dieser Formel für die Thionursäure natürlich, dass bei Anwendung secundärer Amine ein neutrales Sulfitadditionsproduct isolirt werden kann, für das bis jetzt kein Analogon existirt. Denn es kann sich nicht weiter verändern, während es bei Anwendung von Ammoniak und primären Aminen vielleicht auch intermediär entsteht, sich aber sofort unter Wasseraustritt in das entsprechende thiouursaure Salz verwandelt.

21) Schiff, diese Ammalon $210,132$.

2) Beilstein, Handb., 1, 1375. 
Piloty und Finckh, Ueber das Uramil.

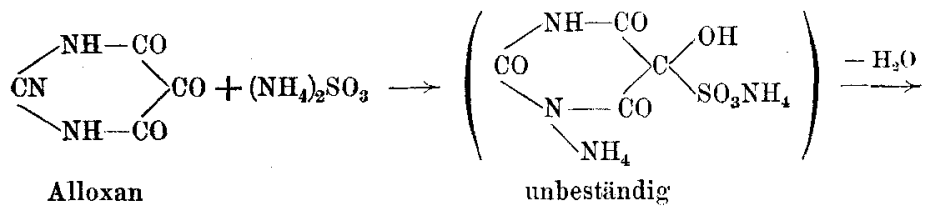<smiles>NO[NH2+][C@H]1[14CH2]NC[14CH2]N[14CH2]1</smiles>

thionursatures Ammon.

(Geschlossen am 9. Mai 1904.) 